\title{
Universe as a domain wall
}

\author{
André Lukas and Burt A. Ovrut \\ Department of Physics, University of Pennsylvania, Philadelphia, Pennsylvania 19104-6396 \\ K. S. Stelle \\ The Blackett Laboratory, Imperial College, London SW7 2BZ, United Kingdom \\ and TH Division, CERN, CH-1211 Geneva 23, Switzerland \\ Daniel Waldram \\ Department of Physics, Joseph Henry Laboratories, Princeton University, Princeton, New Jersey 08544
}

(Received 1 July 1998; published 1 March 1999)

\begin{abstract}
It is shown that the effective five-dimensional theory of the strongly coupled heterotic string is a gauged version of $N=1$ five-dimensional supergravity with four-dimensional boundaries. For the universal supermultiplets, this theory is explicitly constructed by a generalized dimensional reduction procedure on a Calabi-Yau manifold. A crucial ingredient in the reduction is the retention of a "non-zero mode" of the four-form field strength, leading to the gauging of the universal hypermultiplet by the graviphoton. We show that this theory has an exact three-brane domain wall solution which reduces to Witten's "deformed" Calabi-Yau background upon linearization. This solution consists of two parallel three-branes with sources provided by the fourdimensional boundary theories and constitutes the appropriate background for a reduction to four dimensions. Four-dimensional space-time is then identified with the three-brane world volume. [S0556-2821(98)02122-5]
\end{abstract}

PACS number(s): 11.25.Mj, 04.65.+e, 11.27.+d

\section{INTRODUCTION}

The strongly coupled $E_{8} \times E_{8}$ heterotic string has been identified as the 11-dimensional limit of M-theory compactified on an $S^{1} / Z_{2}$ orbifold with a set of $E_{8}$ gauge fields at each ten-dimensional orbifold fixed plane [1,2]. Witten has shown that there exists a consistent compactification of this M-theory limit on a deformed Calabi-Yau three-fold, leading to a supersymmetric $N=1$ theory in four dimensions [3]. Matching at the tree level to the phenomenological gravitational and grand-unified couplings $[3,4]$, one finds that the orbifold must be larger than the Calabi-Yau radius, which is of the order of the 11-dimensional Planck length. This suggests that there is a regime where the universe appears fivedimensional. It is then important to find the five-dimensional effective action, describing the low-energy physics of the strongly coupled heterotic string and which underlies phenomenologically relevant four-dimensional $N=1$ supergravity models. Furthermore, this theory constitutes a new setting for early universe string (M-theory) cosmology, which has traditionally been studied in the framework of the fourdimensional effective action. Although some formal and phenomenological aspects of the strongly coupled heterotic string have been studied in the literature [5-30], a derivation of the five-dimensional effective action from Horava-Witten theory and a detailed discussion of its properties have remained missing. (Some aspects of five-dimensional physics, however, were considered in $[4,24,31,32]$.)

In the present paper, we derive this effective fivedimensional theory for the universal bulk fields, that is, the gravity supermultiplet and the universal hypermultiplet. We shall show that the relevant consistent reduction from 11 to five dimensions on a Calabi-Yau manifold requires the inclusion of non-zero values of the four-form field strength in the internal Calabi-Yau directions. This leads to a gauged fivedimensional supergravity action with a potential term that has not previously been constructed. More precisely, given the universal hypermultiplet coset manifold [33] $\mathcal{M}_{Q}$ $=S U(2,1) / S U(2) \times U(1)$, we find that a subgroup $U(1) \subset S U(2) \times U(1)$ is gauged, with the vector field in the gravity supermultiplet as the corresponding gauge boson. Owing to the potential, flat space is not a solution of this five-dimensional theory without the Calabi-Yau space decompactifying. However, the equations of motion do admit a three-brane solution that preserves half of the remaining $D$ $=5$ supersymmetries where the Calabi-Yau space remains compact. This is supported by source terms on the fixed orbifold planes of the five-dimensional space. This Bogoliubov-Prasad-Sommerfield (BPS) three-brane constitutes the "vacuum" of the five-dimensional theory and it is the appropriate background for a further reduction to fourdimensional $N=1$ supergravity theories. In such a reduction, four-dimensional space-time becomes identified with the three-brane world volume. We will show that the linearized version of this three-brane corresponds to Witten's "deformed" Calabi-Yau solution, which was constructed only to first non-trivial order in powers of the 11-dimensional Newton constant. Thus, our solution represents a generalization of this original background, as it is an exact solution of the effective low energy theory. The inversion of the HoravaWitten construction by first performing a generalized Kaluza-Klein reduction from 11 down to five dimensions, and then finally from five to four dimensions, is more natural for two reasons. First, as noted above, the scale of the fifth dimension is larger than that of the Calabi-Yau manifold. Second, the generalized Kaluza-Klein reduction is a consistent truncation, meaning that, from the point of view of the bulk theory, the heavy Calabi-Yau modes can simply be con- 
sistently set to zero without inducing higher-order corrections. The reduction from five to four dimensions will, however, require carefully integrating out the non-trivial fivedimensional modes, giving rise to higher-order corrections of potential phenomenological interest. The relation between Witten's deformed Calabi-Yau solution and the fivedimensional domain wall solution can also be described using brane language. As we will see, there is a natural interpretation of Witten's solution as a collection of five-branes wrapped on two-cycles of the Calabi-Yau space and lying in the orbifold fixed planes. Reduced to five dimensions, these then become three-branes spanning the orbifold fixed planes.

In summary, we argue that it is a gauged version of fivedimensional supergravity that is the correct arena for considering the effective action of the strongly coupled $E_{8} \times E_{8}$ heterotic string in the intermediate energy range. This effective theory has three-brane domain-wall BPS solutions, with the three-brane world volume corresponding to the orbifold planes. These solutions represent the correct background for making contact with four-dimensional low-energy physics.

Let us now summarize our conventions. We will consider 11-dimensional spacetime compactified on a Calabi-Yau space $X$, with the subsequent reduction down to four dimensions effectively provided by a double-domain-wall background, corresponding to an $S^{1} / Z_{2}$ orbifold. We use coordinates $x^{I}$ with indices $I, J, K, \ldots=0, \ldots, 9,11$ to parametrize the full 11-dimensional space $M_{11}$. Throughout this paper, when we refer to orbifolds, we will work in the "upstairs" picture with the orbifold $S^{1} / Z_{2}$ in the $x^{11}$-direction. We choose the range $x^{11} \in[-\pi \rho, \pi \rho]$ with the end points being identified. The $Z_{2}$ orbifold symmetry acts as $x^{11} \rightarrow-x^{11}$. Then there exist two ten-dimensional hyperplanes fixed under the $Z_{2}$ symmetry which we denote by $M_{10}^{(i)}, i=1,2$. Locally, they are specified by the conditions $x^{11}=0, \pi \rho$. Indices with overbars, $\bar{I}, \bar{J}, \bar{K}, \ldots=0, \ldots, 9$, are used for the ten-dimensional space orthogonal to the orbifold. Upon reduction on the Calabi-Yau space we have a five-dimensional spacetime $M_{5}$ labeled by indices $\alpha, \beta, \gamma, \ldots=0, \ldots, 3,11$. The orbifold fixed planes become four-dimensional with indices $\mu, \nu, \rho, \ldots$ $=0, \ldots, 3$. We use indices $A, B, C, \ldots=4, \ldots, 9$ for the CalabiYau space. The 11-dimensional Dirac-matrices $\Gamma^{I}$ with $\left\{\Gamma^{I}, \Gamma^{J}\right\}=2 g^{I J}$ are decomposed as $\Gamma^{I}=\left\{\gamma^{\alpha} \otimes \lambda, \mathbf{1} \otimes \lambda^{A}\right\}$ where $\gamma^{\alpha}$ and $\lambda^{A}$ are the five- and six-dimensional Dirac matrices, respectively. Here, $\lambda$ is the chiral projection matrix in six dimensions with $\lambda^{2}=1$. Spinors in 11 dimensions will be Majorana spinors with 32 real components throughout the paper. In five dimensions we use symplectic-real spinors [35] $\psi^{i}$ where $i=1,2$ is an $S U(2)$ index, corresponding to the automorphism group of the $N=1$ supersymmetry algebra in five dimensions. We will follow the conventions given in [36]. Fields will be required to have a definite behavior under the $Z_{2}$ orbifold symmetry in $D=11$. We demand a bosonic field $\Phi$ to be even or odd, that is, $\Phi\left(x^{11}\right)= \pm \Phi\left(-x^{11}\right)$. For a spinor $\Psi$ the condition is $\Gamma_{11} \Psi\left(-x^{11}\right)=\Psi\left(x^{11}\right)$ so that the projection to one of the orbifold planes leads to a tendimensional Majorana-Weyl spinor with positive chirality. Similarly, in five dimensions, bosonic fields will be either even or odd. We can choose a basis for the $S U(2)$ automor- phism group such that symplectic-real spinors $\psi^{i}$ satisfy the constraint $\gamma_{11} \psi^{i}\left(-x^{11}\right)=\left(\tau_{3}\right)_{j}^{i} \psi^{j}\left(x^{11}\right)$ where $\tau_{a}$ are the Pauli spin matrices: so $\tau_{3}=\operatorname{diag}(1,-1)$.

\section{STRONGLY COUPLED HETEROTIC STRING AND CALABI-YAU SOLUTIONS}

To set the scene for our later discussion, we will now briefly review the effective description of strongly coupled heterotic string theory as 11-dimensional supergravity with boundaries given by Hořava and Witten $[1,2]$. In addition, we present, in a simple form, the solutions of this theory [3] appropriate for a reduction to $N=1$ theories in four dimensions using the explicit form of these solutions given in Ref. [20].

The bosonic part of the action is of the form

$$
S=S_{\mathrm{SG}}+S_{\mathrm{YM}}
$$

where $S_{\mathrm{SG}}$ is the familiar 11-dimensional supergravity,

$$
\begin{aligned}
S_{\mathrm{SG}}= & -\frac{1}{2 \kappa^{2}} \int_{M^{11}} \sqrt{-g}\left[R+\frac{1}{24} G_{I J K L} G^{I J K L}\right. \\
& \left.+\frac{\sqrt{2}}{1728} \epsilon^{I_{1} \ldots I_{11}} C_{I_{1} I_{2} I_{3}} G_{I_{4} \ldots I_{7}} G_{I_{8}} \ldots I_{11}\right],
\end{aligned}
$$

and $S_{\mathrm{YM}}$ are the two $E_{8}$ Yang-Mills theories on the orbifold planes explicitly given by ${ }^{1}$

$$
\begin{aligned}
S_{\mathrm{YM}}= & -\frac{1}{8 \pi \kappa^{2}}\left(\frac{\kappa}{4 \pi}\right)^{2 / 3} \int_{M_{10}^{(1)}} \sqrt{-g}\left\{\operatorname{tr}\left(F^{(1)}\right)^{2}-\frac{1}{2} \operatorname{tr} R^{2}\right\} \\
& -\frac{1}{8 \pi \kappa^{2}}\left(\frac{\kappa}{4 \pi}\right)^{2 / 3} \int_{M_{10}^{(2)}} \sqrt{-g}\left\{\operatorname{tr}\left(F^{(2)}\right)^{2}-\frac{1}{2} \operatorname{tr} R^{2}\right\} .
\end{aligned}
$$

Here $F_{\overline{I J}}^{(i)}$ are the two $E_{8}$ gauge field strengths and $C_{I J K}$ is the 3 -form with field strength $G_{I J K L}=24 \partial_{[I} C_{J K L]}$. In order for the above theory to be supersymmetric as well as anomaly free, the Bianchi identity for $G$ should receive a correction such that

$$
\begin{aligned}
(d G)_{11 \bar{I} \bar{J} \bar{K} \bar{L}}= & -\frac{1}{2 \sqrt{2} \pi}\left(\frac{\kappa}{4 \pi}\right)^{2 / 3} \\
& \times\left\{J^{(1)} \delta\left(x^{11}\right)+J^{(2)} \delta\left(x^{11}-\pi \rho\right)\right\}_{\overline{I J} \bar{K} \bar{L}}
\end{aligned}
$$

\footnotetext{
${ }^{1} \mathrm{We}$ note that there is a debate in the literature about the precise value of the Yang-Mills coupling constant in terms of $\kappa$. While we quote the original value [2,37] the value found in Ref. [10] is smaller. In the second case, the coefficients in the Yang-Mills action (3) and the Bianchi identity (4) should both be multiplied by $2^{-1 / 3}$. This potential factor will not be essential in the following discussion as it will simply lead to a redefinition of the fivedimensional coupling constants. We will comment on this point later on.
} 
where the sources are given by

$$
J^{(i)}=\operatorname{tr} F^{(i)} \wedge F^{(i)}-\frac{1}{2} \operatorname{tr} R \wedge R .
$$

Under the $Z_{2}$ orbifold symmetry, the field components $g_{\bar{I} \bar{J}}, g_{11,11}, C_{\bar{I} \bar{J} 11}$ are even, while $g_{\bar{I} 11}, C_{\bar{I} \bar{J} \bar{K}}$ are odd. We note that the above boundary actions contain, in addition to the Yang-Mills terms, $\operatorname{tr} R^{2}$ terms which were not part of the original theory derived in [2]. It was argued in Ref. [20] that these terms are required by supersymmetry, since they pair with the $R^{2}$ terms in the Bianchi identity (4) in analogy to the weakly coupled case. The existence of these terms will be of some importance in the following.

One way to view this theory is to draw an analogy between the orbifold planes and D-branes in type II theories. A collection of $\mathrm{D} p$-branes is described by a $U(N)$ gauge theory. The $\mathrm{D} p$-brane charge is measured by $\operatorname{tr} \mathbf{1}=N$, while exciting a $\mathrm{D}(p-2)$-brane charge corresponds to having a non-trivial $\operatorname{tr} F$, a $\mathrm{D}(p-4)$-brane charge corresponds to non-trivial $\operatorname{tr} F \wedge F$ and so on [38]. Similarly, if the original D-branes are on a curved manifold, then there is also an induced charge for lower-dimensional branes given by $\operatorname{tr} R \wedge R$ and higher even powers [39]. Applying this picture to our situation, the role of the $U(N)$ gauge field on the D-brane world volume is here played by the $E_{8}$ gauge fields on the orbifold planes. The correction to the Bianchi identity then has the interpretation of exciting an M5-brane charge in the orbifold plane. In Ref. [13] this picture has been made explicit by constructing a gauge five-brane in this theory.

We would now like to discuss solutions of the above theory which preserve four of the 32 supercharges leading, upon compactification, to four dimensional $N=1$ supergravities. This task is significantly complicated by the fact that the sources in the Bianchi identity (4) are located on the orbifold planes with the gravitational part distributed equally between the two planes. While the standard embedding of the spin connection into the gauge connection

$$
\operatorname{tr} F^{(1)} \wedge F^{(1)}=\operatorname{tr} R \wedge R
$$

leads to vanishing source terms in the weakly coupled heterotic string Bianchi identity (which, in turn, allows one to set the antisymmetric tensor gauge field to zero), in the present case, one is left with non-zero sources $\pm \operatorname{tr} R \wedge R$ on the two hyperplanes. As a result, the antisymmetric tensor field $G$ and, hence, the second term in the gravitino supersymmetry variation,

$$
\delta \Psi_{I}=D_{I} \eta+\frac{\sqrt{2}}{288}\left(\Gamma_{I J K L M}-8 g_{I J} \Gamma_{K L M}\right) G^{J K L M} \eta+\cdots
$$

do not vanish. Thus, straightforwardly compactifying on a Calabi-Yau manifold no longer provides a solution to the Killing spinor equation $\delta \Psi_{I}=0$. The problem can, however, be treated perturbatively in powers of the 11-dimensional Newton constant $\kappa$. To lowest order, one can start with a manifold $X \times S^{1} / Z_{2} \times M_{4}$ where $X$ is a Calabi-Yau three-fold and $M_{4}$ is four-dimensional Minkowski space. This manifold has an $x^{11}$-independent (and hence chiral) Killing spinor $\eta$ which corresponds to four preserved supercharges. Then, one can determine the first order corrections to this background and the spinor $\eta$ so that the gravitino variation vanishes to order $\kappa^{2 / 3}$

The existence of such a distorted background solution to order $\kappa^{2 / 3}$ has been demonstrated in Ref. [3]. To see its explicit form, let us start with the zeroth order metric

$$
d s_{11}^{2}=\eta_{\mu \nu} d x^{\mu} d x^{\nu}+R_{0}^{2}\left(d x^{11}\right)^{2}+V_{0}^{1 / 3} \Omega_{A B} d x^{A} d x^{B},
$$

where $\Omega_{A B}$ is a Calabi-Yau metric with Kähler form $\omega_{a \bar{b}}$ $=i \Omega_{a \bar{b}}$. (Here $a$ and $\bar{b}$ are holomorphic and antiholomorphic indices.) To keep track of the scaling properties of the solution, we have introduced moduli $V_{0}$ and $R_{0}$ for the Calabi-Yau volume and the orbifold radius, respectively. It was shown in [3] that, to order $\kappa^{2 / 3}$, the metric can be written in the form

$$
\begin{aligned}
d s_{11}^{2}= & (1+\hat{b}) \eta_{\mu \nu} d x^{\mu} d x^{\nu}+R_{0}^{2}(1+\hat{\gamma})\left(d x^{11}\right)^{2} \\
& +V_{0}^{1 / 3}\left(\Omega_{A B}+h_{A B}\right) d x^{A} d x^{B}
\end{aligned}
$$

where the functions $\hat{b}, \hat{\gamma}$ and $h_{A B}$ depend on $x^{11}$ and the Calabi-Yau coordinates. Furthermore, as we have discussed, $G_{A B C D}$ and $G_{A B C 11}$ receive a contribution of order $\kappa^{2 / 3}$ from the Bianchi identity source terms. To get the general explicit form of the corrections, one has to solve the relations given in Ref. [3]. This can be done by dualizing the antisymmetric tensor field and using a harmonic expansion on the CalabiYau space [20].

Here, we quote those results simplified in two essential ways. First, we drop all terms corresponding to non-zero eigenvalue harmonics on the Calabi-Yau space. These terms will be of no relevance to the low energy theory, since they correspond to heavy Calabi-Yau modes which decouple at this order. Second, we write only the one massless term that is related to the Calabi-Yau breathing mode. This will be sufficient for all applications dealing only with the universal moduli. Given these simplifications, the corrections are explicitly

$$
\begin{aligned}
\hat{b} & =-\frac{\sqrt{2} R_{0}}{3 V_{0}^{2 / 3}} \alpha\left(\left|x^{11}\right|-\pi \rho / 2\right) \\
\hat{\gamma} & =\frac{2 \sqrt{2} R_{0}}{3 V_{0}^{2 / 3}} \alpha\left(\left|x^{11}\right|-\pi \rho / 2\right) \\
h_{A B} & =\frac{\sqrt{2} R_{0}}{3 V_{0}^{2 / 3}} \alpha\left(\left|x^{11}\right|-\pi \rho / 2\right) \Omega_{A B} \\
G_{A B C D} & =\frac{1}{6} \alpha \epsilon_{A B C D}^{E F} \omega_{E F} \epsilon\left(x^{11}\right)
\end{aligned}
$$$$
G_{A B C 11}=0
$$

with 


$$
\begin{aligned}
& \alpha=-\frac{1}{8 \sqrt{2} \pi v}\left(\frac{\kappa}{4 \pi}\right)^{2 / 3} \int_{X} \omega \wedge \operatorname{tr} R^{(\Omega)} \wedge R^{(\Omega)}, \\
& v=\int_{X} \sqrt{\Omega} .
\end{aligned}
$$

Here $\epsilon\left(x^{11}\right)$ is the step function which is $+1(-1)$ for $x^{11}$ positive (negative). Note that, by dropping the massive modes, these expressions take a very simple form representing a linear increase of the corrections along the orbifold. Even more significantly, and unlike the exact solution including the heavy modes, the above approximation leads to a corrected metric $\Omega_{A B}+h_{A B}$ that is still of Calabi-Yau type at each point on the $S^{1} / Z_{2}$ orbifold. The Calabi-Yau volume (and, if all moduli are included, also its shape), however, is continuously changing across the orbifold. More generally, one can think of the internal part of the corrected metric as a curve in the Calabi-Yau moduli space.

Returning to the D-brane perspective, one can view the above configuration as the linearized solution for a collection of five-branes embedded in the orbifold planes. The relation (6) fixes equal amounts of five-brane charge, $\frac{1}{2} \operatorname{tr} R \wedge R$, on each orbifold fixed plane, where the five-branes are confined to reside. Since $\operatorname{tr} R \wedge R \in H^{2,2}(X)$, we can associate a different five-brane charge for each independent element of $H^{2,2}(X)$. The five-branes themselves are associated with Poincare dual cycles. Thus they span the non-compact fourdimensional space together with a two-cycle in the CalabiYau space. In particular, from the five-dimensional point of view, they are three-branes localized on the orbifold planes. Witten's construction ensures that this configuration of branes preserves one-eighth of the supersymmetry. Finally, restricting to just the Calabi-Yau breathing modes corresponds to keeping only the five-brane which spans the holomorphic two-cycle in the Calabi-Yau space defined by the Kähler form.

\section{FIVE-DIMENSIONAL EFFECTIVE ACTION}

Phenomenologically, there is a regime where the universe appears five-dimensional. We would, therefore, like to derive an effective theory in the space consisting of the usual four space-time dimensions and the orbifold, based on the background solution discussed in the previous section. As we have already mentioned, we will consider universal zero modes only, that is, the five-dimensional graviton supermultiplet and the breathing mode of the Calabi-Yau space, along with its superpartners. These form a hypermultiplet in five dimensions. Furthermore, to keep the discussion as simple as possible, we will not consider boundary gauge matter fields. This simple framework suffices to illustrate our main ideas. The general case will be presented elsewhere [40].

Naively, one might attempt to perform the actual reduction directly on the background given in Eqs. (9) and (10)(14). This would, however, lead to a complicated fivedimensional theory with explicit $x^{11}$-dependence in the action. Moreover, this background preserves only four supercharges whereas the minimal supergravity in five dimensions
$(N=1)$ is invariant under twice this amount of supersymmetry.

A useful observation here is that, since we retain the dependence on the orbifold coordinate, we can actually absorb the metric deformations in Eqs. (9) and (10)-(14) into the five-dimensional metric moduli. That is, the $x^{11}$-dependent scale factors $\hat{b}$ and $\hat{\gamma}$ of the four-dimensional space and of the orbifold can be absorbed into the five-dimensional (Einstein frame) metric $g_{\alpha \beta}$ while, analogously, the variation of the Calabi-Yau volume along the orbifold encoded in $h_{A B}$ can be absorbed into a modulus $V .^{2}$ More precisely, we can perform the Kaluza-Klein reduction on the metric

$$
d s_{11}^{2}=V^{-2 / 3} g_{\alpha \beta} d x^{\alpha} d x^{\beta}+V^{1 / 3} \Omega_{A B} d x^{A} d x^{B} .
$$

This rewriting suggests a change of perspective: rather than reducing on the Witten vacuum, we can try to find an effective five-dimensional theory where we recover the Witten vacuum as a particular solution.

We see that, since we have absorbed the deformation into the moduli, the background corresponding to the metric (16) preserves eight supercharges, the appropriate number for a reduction down to five dimensions. It might appear that we are simply performing a standard reduction of 11dimensional supergravity on a Calabi-Yau space to five dimensions, for example, in the way described in Ref. [41]. If this were the case, then it would be hard to understand how the resulting five-dimensional theory could encode any information about the deformed Calabi-Yau background. There are, however, two important ingredients that we have not yet included. One is obviously the existence of the boundary theories. We will return to this point shortly. First, however, let us explain a somewhat unconventional addition to the bulk theory that must be included.

Although we could absorb all metric corrections into the five-dimensional metric moduli, the same is not true for the 4-form field. Specifically, for the nonvanishing component $G_{A B C D}$ in Eq. (13) there is no corresponding zero mode field. ${ }^{3}$ Therefore, in the reduction, we should take this part of $G$ explicitly into account. In the terminology of Ref. [42], such an antisymmetric tensor field configuration is called a "'non-zero mode.' More generally, a non-zero mode is a background antisymmetric tensor field that solves the equations of motion but, unlike antisymmetric tensor field moduli, has nonvanishing field strength. Such configurations, for a $p$-form field strength, can be identified with the cohomology group $H^{p}(M)$ of the manifold $M$ and, in par-

\footnotetext{
${ }^{2}$ Note that we could not apply a similar method for a reduction down to four dimensions, as all moduli fields would then be $x^{11}$ independent. In this case, one should work with the background in the form (9), (10)-(14) as done in Ref. [20].

${ }^{3}$ This can be seen from the mixed part of the Bianchi identity $\partial_{\alpha} G_{A B C D}=0$ which shows that the constant $\alpha$ in Eqs. (10)-(14) cannot be promoted as stands to a five-dimensional field. It is possible to dualize in five dimensions, so that the constant $\alpha$ is promoted to a five-form field, but we will not pursue this formulation here.
} 
ticular, exist if this cohomology group is nontrivial. In the case under consideration, the relevant cohomology group is $H^{4}(X)$ which is nontrivial for a Calabi-Yau manifold $X$ since $h^{2,2}=h^{1,1} \geqslant 1$. Again, the form of $G_{A B C D}$ in Eq. (13) is somewhat special, reflecting the fact that we are concentrating here on the universal moduli. In the general case, $G_{A B C D}$ would be a linear combination of all harmonic $(2,2)$-forms.

The complete configuration for the antisymmetric tensor field that we use in the reduction is given by

$$
\begin{gathered}
C_{\alpha \beta \gamma}, \quad G_{\alpha \beta \gamma \delta}=24 \partial_{[\alpha} C_{\beta \gamma \delta]} \\
C_{\alpha A B}=\frac{1}{6} \mathcal{A}_{\alpha} \omega_{A B}, \quad G_{\alpha \beta A B}=\mathcal{F}_{\alpha \beta} \omega_{A B}=2 \partial_{[\alpha} \mathcal{A}_{\beta]} \omega_{A B}, \\
C_{A B C}=\frac{1}{6} \xi \omega_{A B C}+\frac{1}{6} \bar{\omega}_{A B C}, \quad G_{\alpha A B C}=\partial_{\alpha} \xi \omega_{A B C}+\partial_{\alpha} \bar{\omega}_{A B C}
\end{gathered}
$$

and the non-zero mode is

$$
G_{A B C D}=\frac{\alpha}{6} \epsilon_{A B C D}^{E F} \omega_{E F} \epsilon\left(x^{11}\right),
$$

where $\alpha$ was defined in Eq. (15). Here, $\omega_{A B C}$ is the harmonic $(3,0)$ form on the Calabi-Yau space and $\xi$ is the corresponding (complex) scalar zero mode. In addition, we have a fivedimensional vector field $\mathcal{A}_{\alpha}$ and 3-form $C_{\alpha \beta \gamma}$, which can be dualized to a scalar $\sigma$. The total bulk field content of the five-dimensional theory is then given by the gravity multiplet $\left(g_{\alpha \beta}, \mathcal{A}_{\alpha}, \psi_{\alpha}^{i}\right)$ together with the universal hypermultiplet $\left(V, \sigma, \xi, \bar{\xi}, \zeta^{i}\right)$ where $\psi_{\alpha}^{i}$ and $\zeta^{i}$ are the gravitini and the hypermultiplet fermions, respectively, and $i=1,2$. From their relations to the 11-dimensional fields, it is easy to see that $g_{\mu \nu}, g_{11,11}, \mathcal{A}_{11}, \sigma$ must be even under the $Z_{2}$ action whereas $g_{\mu 11}, \mathcal{A}_{\mu}, \xi$ must be odd.

Examples of compactifications with non-zero modes in pure 11-dimensional supergravity on various manifolds including Calabi-Yau three-folds have been studied in Ref. [43]. There is, however, one important way in which our non-zero mode differs from other non-zero modes in pure 11-dimensional supergravity. Whereas the latter may be viewed as an optional feature of generalized Kaluza-Klein reduction, the non-zero mode in Horava-Witten theory that we have identified cannot be turned off. This can be seen from the fact that the constant $\alpha$ in expression (18) cannot be set to zero, unlike the case in pure 11-dimensional supergravity where it would be arbitrary, since it is fixed by Eq. (15) in terms of Calabi-Yau data. This fact is, of course, intimately related to the existence of the boundary source terms, particularly in the Bianchi identity (4). As we will see, keeping the non-zero mode in the derivation of the fivedimensional action is crucial to finding a solution of this theory that corresponds to the deformed Calabi-Yau space discussed in the previous section.

Let us now turn to a discussion of the boundary theories. In the five-dimensional space $M_{5}$ of the reduced theory, the orbifold fixed planes constitute four-dimensional hypersurfaces which we denote by $M_{4}^{(i)}, i=1,2$. Clearly, since we have used the standard embedding, there will be an $E_{6}$ gauge field $A_{\mu}^{(1)}$ accompanied by gauginos and gauge matter fields on the orbifold plane $M_{4}^{(1)}$. For simplicity, we will set these gauge matter fields to zero in the following. The field content of the orbifold plane $M_{4}^{(2)}$ consists of an $E_{8}$ gauge field $A_{\mu}^{(2)}$ and the corresponding gauginos. In addition, there is another important boundary effect which results from the non-zero internal gauge field and gravity curvatures. More precisely, note that

$$
\begin{aligned}
\int_{X} \sqrt{\Omega} \operatorname{tr} F_{A B}^{(1)} F^{(1) A B} & =\int_{X} \sqrt{\Omega} \operatorname{tr} R_{A B} R^{A B} \\
& =-16 \sqrt{2} \pi v\left(\frac{4 \pi}{\kappa}\right)^{2 / 3} \alpha, \\
F_{A B}^{(2)} & =0 .
\end{aligned}
$$

In view of the boundary actions (3), it follows that we will retain cosmological type terms with opposite signs on the two boundaries. Note that the size of those terms is set by the same constant $\alpha$, given by Eq. (15), which determines the magnitude of the non-zero mode. The boundary cosmological terms are another important ingredient in reproducing the 11-dimensional background as a solution of the fivedimensional theory.

We can now compute the five-dimensional effective action of Horava-Witten theory. Using the field configuration (16) -(19) we find from the action (1)-(3) that

$$
S_{5}=S_{\text {grav }}+S_{\text {hyper }}+S_{\text {bound }}
$$

where

$$
\begin{aligned}
S_{\text {grav }}= & -\frac{1}{2 \kappa_{5}^{2}} \int_{M_{5}} \sqrt{-g}\left[R+\frac{3}{2} \mathcal{F}_{\alpha \beta} \mathcal{F}^{\alpha \beta}+\frac{1}{\sqrt{2}} \epsilon^{\alpha \beta \gamma \delta \epsilon} \mathcal{A}_{\alpha} \mathcal{F}_{\beta \gamma} \mathcal{F}_{\delta \epsilon}\right] \\
S_{\text {hyper }}= & -\frac{1}{2 \kappa_{5}^{2}} \int_{M_{5}} \sqrt{-g}\left[\frac{1}{2 V^{2}} \partial_{\alpha} V \partial^{\alpha} V+\frac{2}{V} \partial_{\alpha} \xi \partial^{\alpha} \bar{\xi}+\frac{V^{2}}{24} G_{\alpha \beta \gamma \delta} G^{\alpha \beta \gamma \delta}\right. \\
& \left.+\frac{\sqrt{2}}{24} \epsilon^{\alpha \beta \gamma \delta \epsilon} G_{\alpha \beta \gamma \delta}\left[i\left(\xi \partial_{\epsilon} \bar{\xi}-\bar{\xi} \partial_{\epsilon} \xi\right)+2 \alpha \epsilon\left(x^{11}\right) \mathcal{A}_{\epsilon}\right]+\frac{1}{3 V^{2}} \alpha^{2}\right]
\end{aligned}
$$




$$
S_{\text {bound }}=\frac{\sqrt{2}}{\kappa_{5}^{2}} \int_{M_{4}^{(1)}} \sqrt{-g} V^{-1} \alpha-\frac{\sqrt{2}}{\kappa_{5}^{2}} \int_{M_{4}^{(2)}} \sqrt{-g} V^{-1} \alpha-\frac{1}{16 \pi \alpha_{\mathrm{GUT}}} \sum_{i=1}^{2} \int_{M_{4}^{(i)}} \sqrt{-g} V \operatorname{tr} F_{\mu \nu}^{(i) 2}
$$

In this expression, we have now dropped higher-derivative terms. The four-form field strength $G_{\alpha \beta \gamma \delta}$ is subject to the Bianchi identity

$$
\begin{aligned}
(d G)_{11 \mu \nu \rho \sigma}= & -\frac{\kappa_{5}^{2}}{4 \sqrt{2} \pi \alpha_{\mathrm{GUT}}} \\
& \times\left\{J^{(1)} \delta\left(x^{11}\right)+J^{(2)} \delta\left(x^{11}-\pi \rho\right)\right\}_{\mu \nu \rho \sigma}
\end{aligned}
$$

which follows directly from the 11-dimensional Bianchi identity (4). The currents $J^{(i)}$ have been defined in Eq. (5). The five-dimensional Newton constant $\kappa_{5}$ and the YangMills coupling $\alpha_{\mathrm{GUT}}$ are expressed in terms of 11dimensional quantities as ${ }^{4}$

$$
\kappa_{5}^{2}=\frac{\kappa^{2}}{v}, \quad \alpha_{\mathrm{GUT}}=\frac{\kappa^{2}}{2 v}\left(\frac{4 \pi}{\kappa}\right)^{2 / 3} .
$$

We have checked the consistency of the truncation which leads to the above action by an explicit reduction of the 11-dimensional equations of motion to five dimensions. Note that the potential terms in the bulk and on the boundaries arise precisely from the inclusion of the non-zero mode and the gauge and gravity field strengths, respectively. Since we have compactified on a Calabi-Yau space, we expect the bulk part of the above action to have eight preserved supercharges and, therefore, to correspond to minimal $N=1$ supergravity in five dimensions. Accordingly, let us compare the result (21)-(23) to the known $N=1$ supergravity-matter theories in five dimensions $[34,36,44,45]$.

In these theories, the scalar fields in the universal hypermultiplet parametrize a quaternionic manifold with coset structure $\mathcal{M}_{Q}=S U(2,1) / S U(2) \times U(1)$. Hence, to compare our action to these we should dualize the three-form $C_{\alpha \beta \gamma}$ to a scalar field $\sigma$ by setting (in the bulk)

$$
G_{\alpha \beta \gamma \delta}=\frac{1}{\sqrt{2} V^{2}} \epsilon_{\alpha \beta \gamma \delta \epsilon}\left[\partial^{\epsilon} \sigma-i\left(\xi \partial^{\epsilon} \bar{\xi}-\bar{\xi} \partial^{\epsilon} \xi\right)-2 \alpha \epsilon\left(x^{11}\right) \mathcal{A}^{\epsilon}\right] .
$$

Then the hypermultiplet part of the action (22) can be written as

\footnotetext{
${ }^{4}$ The following relations are given for the normalization of the 11-dimensional action as in Eq. (1). If instead the normalization of [10] is used, the expression for $\alpha_{\mathrm{GUT}}$ gets rescaled to $\alpha_{\mathrm{GUT}}$ $=2^{1 / 3}\left(\kappa^{2} / 2 v\right)(4 \pi / \kappa)^{2 / 3}$. Otherwise the action and Bianchi identities are unchanged, except that in the expression (19) for $\alpha$ the right-hand side is multiplied by $2^{1 / 3}$.
}

$$
S_{\text {hyper }}=-\frac{v}{2 \kappa^{2}} \int_{M_{5}} \sqrt{-g}\left[h_{u v} \nabla_{\alpha} q^{u} \nabla^{\alpha} q^{v}+\frac{1}{3} V^{-2} \alpha^{2}\right]
$$

where $q^{u}=(V, \sigma, \xi, \bar{\xi})$. The covariant derivative $\nabla_{\alpha}$ is defined as $\nabla_{\alpha} q^{u}=\partial_{\alpha} q^{u}+\alpha \epsilon\left(x^{11}\right) \mathcal{A}_{\alpha} k^{u}$ with $k^{u}=(0,-2,0,0)$. The sigma model metric $h_{u v}=\partial_{u} \partial_{v} K_{Q}$ can be computed from the Kähler potential

$$
K_{Q}=-\ln (S+\bar{S}-2 C \bar{C}), \quad S=V+\xi \bar{\xi}+i \sigma, \quad C=\xi .
$$

Consequently, the hypermultiplet scalars $q^{u}$ parametrize a Kähler manifold with metric $h_{u v}$. It can be demonstrated that $k^{u}$ is a Killing vector on this manifold. Using the expressions given in Ref. [46], one can show that this manifold is quaternionic with coset structure $\mathcal{M}_{Q}$. Hence, the terms in Eq. (27) that are independent of $\alpha$ describe the known form of the universal hypermultiplet action. How do we interpret the extra terms in the hypermultiplet action depending on $\alpha$ ? A hint is provided by the fact that one of these $\alpha$-dependent terms modifies the flat derivative in the kinetic energy to a generalized derivative $\nabla_{\alpha}$. This is exactly the combination that we would need if one wanted to gauge the $U(1)$ symmetry on $\mathcal{M}_{Q}$ corresponding to the Killing vector $k^{u}$, using the gauge field $\mathcal{A}_{\alpha}$ in the gravity supermultiplet. In fact, investigation of the other terms in the action, including the fermions, shows that the resulting five-dimensional theory is precisely a gauged form of supergravity. Not only is a $U(1)$ isometry of $\mathcal{M}_{Q}$ gauged, but at the same time a $U(1)$ subgroup of the $S U(2)$ automorphism group is also gauged.

What about the remaining $\alpha$-dependent potential term in the hypermultiplet action? From $D=4, N=2$ theories, we are used to the idea that gauging a symmetry of the quaternionic manifold describing hypermultiplets generically introduces potential terms into the action when supersymmetry is preserved (see for instance [47]). Such potential terms can be thought of as the generalization of pure Fayet-Iliopoulos (FI) terms. This is precisely what happens in our theory as well, with the gauging of the $U(1)$ subgroup inducing the $\alpha$ dependent potential term in Eq. (27). The general gauged action will be discussed in more detail in [40]. Certain pure FI terms were previously considered in [44], but, to our knowledge, such a theory with general gauging has not been constructed previously in five dimensions.

The phenomenon that the inclusion of non-zero modes leads to gauged supergravity theories has already been observed in type II Calabi-Yau compactifications $[48,49]$, while the observation that the vacua of gauged theories correspond to dimensional reduction with non-trivial form-fields has a long history. Recent results relating to intersecting branes are described in [50]. From the form of the Killing vector, we 
see that it is only the scalar field $\sigma$, dual to the four-form $G_{\alpha \beta \gamma \delta}$, which is charged under the $U(1)$ symmetry. Its charge is fixed by $\alpha$. We note that this charge is quantized since, suitably normalized, $\operatorname{tr} R \wedge R$ is an element of $H^{2,2}(X, \mathbf{Z})$. In the brane description of the theory, this is a reflection of the fact that the five-brane charge is quantized.

To analyze the supersymmetry properties of the solutions shortly to be discussed, we need the supersymmetry variations of the fermions associated with the theory (20). They can be obtained either by a reduction of the 11-dimensional gravitino variation (7) or by generalizing the known fivedimensional transformations $[36,45]$ by matching onto gauged four-dimensional $N=2$ theories. It is sufficient for our purposes to keep the bosonic terms only. Both approaches lead to

$$
\begin{aligned}
\delta \psi_{\alpha}^{i}= & D_{\alpha} \epsilon^{i}+\frac{\sqrt{2} i}{8}\left(\gamma_{\alpha}{ }^{\beta \gamma}-4 \delta_{\alpha}^{\beta} \gamma^{\gamma}\right) \mathcal{F}_{\beta \gamma} \epsilon^{i} \\
& -\frac{1}{2} V^{-1 / 2}\left[\partial_{\alpha} \xi\left(\tau_{1}-i \tau_{2}\right)_{j}^{i}-\partial_{\alpha} \bar{\xi}\left(\tau_{1}+i \tau_{2}\right)_{j}^{i}\right] \epsilon^{j} \\
& -\frac{\sqrt{2} i}{96} V \epsilon_{\alpha}{ }^{\beta \gamma \delta \epsilon} G_{\beta \gamma \delta \epsilon}\left(\tau_{3}\right)_{j}^{i} \epsilon^{j} \\
& -\frac{\sqrt{2}}{12} \alpha V^{-1} \epsilon\left(x^{11}\right) \gamma_{\alpha}\left(\tau_{3}\right)_{j}^{i} \epsilon^{j} \\
\delta \zeta^{i}= & \frac{\sqrt{2}}{48} V \epsilon^{\alpha \beta \gamma \delta \epsilon} G_{\alpha \beta \gamma \delta} \gamma_{\epsilon} \epsilon^{i} \\
& -\frac{i}{2} V^{-1 / 2} \gamma^{\alpha}\left[\partial_{\alpha} \xi\left(\tau_{1}-i \tau_{2}\right)^{i}+\partial_{\alpha} \bar{\xi}\left(\tau_{1}+i \tau_{2}\right)_{j}^{i}\right] \epsilon^{j} \\
& +\frac{i}{2} V^{-1} \gamma_{\beta} \partial^{\beta} V \epsilon^{i}-\frac{i}{\sqrt{2}} \alpha V^{-1} \epsilon\left(x^{11}\right)\left(\tau_{3}\right)_{j}^{i} \epsilon^{j} \quad \text { (29) }
\end{aligned}
$$

where $\tau_{i}$ are the Pauli spin matrices.

In summary, we see that the relevant five-dimensional effective theory for the reduction of Horava-Witten theory is a gauged $N=1$ supergravity theory with bulk and boundary potentials. While we have calculated the theory only to order $\kappa^{2 / 3}$, one would expect that M-theory corrections can be described in the same type of theory. For this reason, it would be very desirable to construct the most general gauged fivedimensional $N=1$ supergravity theory coupled to general $N=1$ four-dimensional boundary theories with vector and chiral multiplets [40]. In the context of global supersymmetry, such boundary theories in five dimensions have been studied in Ref. [31]. In this paper, we content ourselves with having identified some of the crucial generalizations that would be required.

\section{DOMAIN-WALL SOLUTION}

Let us recapitulate what we have done so far. To arrive at a simple form for the five-dimensional effective action, we have absorbed the deformation of the Calabi-Yau background metric into the five-dimensional moduli. Effectively, we could then carry out the reduction on a Calabi-Yau space but had to explicitly keep the antisymmetric tensor part of the background as a non-zero mode in the reduction. As a consequence, although Witten's original background preserved only four supercharges, the effective bulk theory has twice that number of preserved supercharges, corresponding to minimal $N=1$ supergravity in five dimensions. For consistency, we should now be able to find the deformations of the Calabi-Yau background as solutions of the effective fivedimensional theory. These solutions should break half the supersymmetry of the five-dimensional bulk theory and preserve Poincaré invariance in four dimensions. Hence, we expect there to be a three-brane domain wall in five dimensions with a world volume lying in the four uncompactified directions. This domain wall can be viewed as the "vacuum" of the five-dimensional theory, in the sense that it provides the appropriate background for a reduction to the $D=4, N=1$ effective theory.

This expectation is made stronger if we recall the brane picture of Witten's background. We argued that this could be described by five-branes with equal amounts of five-brane charge residing on the orbifold planes. From the fivedimensional perspective, the five-branes appear as threebranes residing on the orbifold fixed planes. Thus, in five dimensions, Witten's background must correspond to a pair of parallel three-branes.

We notice that the theory (20) has all of the prerequisites necessary for such a three-brane solution to exist. Generally, in order to have a $(D-2)$-brane in a $D$-dimensional theory, one needs to have a $(D-1)$-form field or, equivalently, a cosmological constant. This is familiar from the eight-brane [51] in massive type IIA supergravity in ten dimensions [52], and has been systematically studied for theories in arbitrary dimension obtained by generalized (Scherk-Schwarz) dimensional reduction [53]. In our case, this cosmological term is provided by the bulk potential term in the action (20). From the viewpoint of the bulk theory, we could have multiple three-brane solutions with an arbitrary number of parallel branes located at various places in the $x^{11}$ direction. As is well known, however, elementary brane solutions have singularities at the location of the branes, needing to be supported by source terms. Natural candidates for those source terms, in our case, are the boundary actions. Given the anomaly-cancellation requirements, this restricts the possible solutions to those representing a pair of parallel three-branes corresponding to the orbifold planes.

From the above discussion, it is clear that in order to find a three-brane solution, we should start with the ansatz

$$
d s_{5}^{2}=a(y)^{2} d x^{\mu} d x^{\nu} \eta_{\mu \nu}+b(y)^{2} d y^{2}
$$

$$
V=V(y)
$$

where $a$ and $b$ are functions of $y=x^{11}$ and all other fields vanish. The general solution for this ansatz, satisfying the equations of motion derived from action (20), is given by 


$$
\begin{aligned}
& a=a_{0} H^{1 / 2} \\
& b=b_{0} H^{2}, \quad H=\frac{\sqrt{2}}{3} \alpha|y|+c_{0} \\
& V=b_{0} H^{3}
\end{aligned}
$$

where $a_{0}, b_{0}$ and $c_{0}$ are constants. We note that the boundary source terms have fixed the form of the harmonic function $H$ in the above solution. Without specific information about the sources, the function $H$ would generically be glued together from an arbitrary number of linear pieces with slopes $( \pm \sqrt{2} / 3) \alpha$. The edges of each piece would then indicate the location of the source terms. The necessity of matching the boundary sources at $y=0$ and $\pi \rho$, however, has forced us to consider only two such linear pieces, namely $y$ $\in[0, \pi \rho]$ and $y \in[-\pi \rho, 0]$. These pieces are glued together at $y=0$ and $\pi \rho$ (recall here that we have identified $\pi \rho$ and $-\pi \rho)$. Therefore, we have

$$
\partial_{y}^{2} H=\frac{2 \sqrt{2}}{3} \alpha[\delta(y)-\delta(y-\pi \rho)]
$$

which shows that the solution represents two parallel threebranes located at the orbifold planes.

We stress that this solution solves the five-dimensional theory (20) exactly, whereas the original deformed CalabiYau solution was only an approximation to order $\kappa^{2 / 3}$. It is straightforward to show that the linearized version of Eq. (31), that is, the expansion to first order in $\alpha=O\left(\kappa^{2 / 3}\right)$, coincides with Witten's solution (9), (10)-(14) upon appropriate matching of the integration constants. Hence, we have found an exact generalization, good to all orders in $\kappa$, of the linearized 11-dimensional solution.

Of course, we still have to check that our solution preserves half of the supersymmetries. When $g_{\alpha \beta}$ and $V$ are the only non-zero fields, the supersymmetry transformations (29) simplify to

$$
\begin{aligned}
\delta \psi_{\alpha}^{i} & =D_{\alpha} \epsilon^{i}-\frac{\sqrt{2}}{12} \alpha \epsilon(y) V^{-1} \gamma_{\alpha}\left(\tau_{3}\right)_{j}^{i} \epsilon^{j} \\
\delta \zeta^{i} & =\frac{i}{2} V^{-1} \gamma_{\beta} \partial^{\beta} V \epsilon^{i}-\frac{i}{\sqrt{2}} \alpha \epsilon(y) V^{-1}\left(\tau_{3}\right)_{j}^{i} \epsilon^{j} .
\end{aligned}
$$

The Killing spinor equations $\delta \psi_{\alpha}^{i}=0, \delta \zeta^{i}=0$ are satisfied for the solution (31) if we require that the spinor $\epsilon^{i}$ be given by

$$
\epsilon^{i}=H^{1 / 4} \epsilon_{0}^{i}, \quad \gamma_{11} \epsilon_{0}^{i}=\left(\tau_{3}\right)_{j}^{i} \epsilon_{0}^{j}
$$

where $\epsilon_{0}^{i}$ is a constant symplectic Majorana spinor. This shows that we have indeed found a BPS solution preserving four of the eight bulk supercharges.

Let us discuss the meaning of this solution in some detail. First, we notice that it fits the general scheme of domain wall solutions in various dimensions. ${ }^{5}$ It is, however, a new solution to the gauged supergravity action (20) in five dimensions which has not been constructed previously. In addition, its source terms are naturally provided by the boundary actions resulting from Hořava-Witten theory. Most importantly, it constitutes the fundamental vacuum solution of a phenomenologically relevant theory. The two parallel threebranes of the solution, separated by the bulk, are oriented in the four uncompactified space-time dimensions, and carry the physical low-energy gauge and matter fields. Therefore, from the low-energy point of view where the orbifold is not resolved the three-brane world volume is identified with four-dimensional space-time. In this sense the Universe resides on the world volume of a three-brane.

Although we have found an exact solution to the (lowest order) low energy theory, thereby improving previous results, it is not clear whether the solution will be exact in the full theory. Strominger [46] has argued that the all-loop corrections (corresponding to corrections to the effective action proportional to powers of $\kappa^{4 / 3} / V$, in our notation) to the quaternionic metric of the universal hypermultiplet can be actually absorbed into a shift of $V$, so that the metric is unchanged. This implies that our solution would be unaffected by such corrections. On the other hand, we have no general argument why the solution should be protected against corrections from higher derivative terms.

In any case, we believe that pursuing the construction of five-dimensional gauged supergravities with boundaries, and the analysis of their soliton structure, in the way indicated in this paper might provide important insights into low energy particle phenomenology as well as early universe cosmology.

\section{ACKNOWLEDGMENTS}

A.L. is supported in part by the Deutsche Forschungsgemeinschaft DFG. A.L. and B.A.O. are supported in part by the DOE under contract No. DE-AC02-76-ER-03071. D.W. is supported in part by the DOE under contract No. DEFG02-91ER40671.

\footnotetext{
${ }^{5}$ In the notation of Ref. [53], it corresponds to choosing $D=5$, $\Delta=4 / 3$ and $a(5)=2$.
}

[1] P. Hořava and E. Witten, Nucl. Phys. B460, 506 (1996).

[2] P. Hořava and E. Witten, Nucl. Phys. B475, 94 (1996).

[3] E. Witten, Nucl. Phys. B471, 135 (1996).

[4] T. Banks and M. Dine, Nucl. Phys. B479, 173 (1996).
[5] P. Hořava, Phys. Rev. D 54, 7561 (1996).

[6] I. Antoniadis and M. Quirós, Phys. Lett. B 392, 61 (1997).

[7] E. Caceres, V. S. Kaplunovsky and I. M. Mandelberg, Nucl. Phys. B493, 73 (1997). 
[8] H.-P. Nilles and S. Stieberger, Nucl. Phys. B499, 3 (1997).

[9] T. Li, J. L. Lopez and D. V. Nanopoulos, Mod. Phys. Lett. A 12, 2647 (1997).

[10] J. O. Conrad, Phys. Lett. B 421, 119 (1998).

[11] E. Dudas and C. Grojean, Nucl. Phys. B507, 553 (1997).

[12] E. Dudas and J. Mourad, Phys. Lett. B 400, 71 (1997).

[13] Z. Lalak, A. Lukas and B. A. Ovrut, Phys. Lett. B 425, 59 (1998).

[14] K. Choi, Phys. Rev. D 56, 6588 (1997).

[15] H. P. Nilles, M. Olechowski and M. Yamaguchi, Phys. Lett. B 415, 24 (1997).

[16] I. Antoniadis and M. Quirós, Phys. Lett. B 416, 327 (1998).

[17] Z. Lalak and S. Thomas, Nucl. Phys. B515, 55 (1998).

[18] E. Dudas, Phys. Lett. B 416, 309 (1998).

[19] J. Ellis, A. E. Faraggi and D. V. Nanopoulos, Phys. Lett. B 419, 123 (1998).

[20] A. Lukas, B. A. Ovrut and D. Waldram, Nucl. Phys. B532, 43 (1998).

[21] D. V. Nanopoulos, "M Phenomenology," in Beyond the Desert, Proceedings, Tegernsee, Germany, 1997 (Institute of Physics, Bristol, 1998), pp. 636-654.

[22] K. Choi, H. B. Kim and C. Muñoz, Phys. Rev. D 57, 7521 (1998).

[23] A. Lukas, B. A. Ovrut and D. Waldram, Phys. Rev. D 57, 7529 (1998).

[24] H. P. Nilles, M. Olechowski and M. Yamaguchi, Nucl. Phys. B530, 43 (1998).

[25] A. Lukas, B. A. Ovrut and D. Waldram, "The Tendimensional Effective Action of Strongly Coupled Heterotic String Theory,"' Report No. UPR-789-T, hep-th/9801087.

[26] T. Li, Phys. Rev. D 57, 7539 (1998).

[27] M. Faux, 'New Consistent Limits of M Theory,' Report No. HUB-EP-98-9, hep-th/9801204.

[28] P. Majumdar and S. SenGupta, Phys. Lett. B 445, 129 (1998).

[29] N. Wyllard, J. High Energy Phys. 04, 009 (1998).

[30] D. Bailin, G. V. Kraniotis and A. Love, Phys. Lett. B 432, 90 (1998).

[31] E. Sharpe, Nucl. Phys. B523, 211 (1998).

[32] E. A. Mirabelli and M. E. Peskin, Phys. Rev. D 58, 065002 (1998).
[33] S. Cecotti, S. Ferrara and L. Girardello, Phys. Lett. B 213, 443 (1988); Int. J. Mod. Phys. A 4, 2475 (1989).

[34] A. H. Chamseddine and H. Nicolai, Phys. Lett. 96B, 89 (1980).

[35] E. Cremmer, in Superspace and Supergravity, edited by S. W. Hawking and M. Rocek (Cambridge University Press, Cambridge, England, 1981), pp. 267-282.

[36] M. Günaydin, G. Sierra and P. K. Townsend, Nucl. Phys. B242, 244 (1084).

[37] S. P. de Alwis, Phys. Lett. B 388, 291 (1996); 392, 332 (1997).

[38] M. Li, Nucl. Phys. B460, 351 (1996).

[39] M. Green, J. A. Harvey and G. Moore, Class. Quantum Grav. 14, 47 (1997).

[40] A. Lukas, B. A. Ovrut, K. S. Stelle and D. Waldram, "Heterotic M Theory in Five Dimensions,'” Report No. UPR-804T, hep-th/9806051.

[41] A. C. Cadavid, A. Ceresole, R. D'Auria and S. Ferrara, Phys. Lett. B 357, 76 (1995); I. Antoniadis, S. Ferrara and T. R. Taylor, Nucl. Phys. B460, 489 (1996).

[42] M. B. Green, J. H. Schwarz and E. Witten, Superstring Theory (Cambridge University Press, Cambridge, England, 1987), Vol. 2, p. 386.

[43] I. V. Lavrinenko, H. Lü and C. N. Pope, Nucl. Phys. B492, 278 (1996).

[44] M. Günaydin, G. Sierra and P. K. Townsend, Nucl. Phys. B253, 573 (1985).

[45] G. Sierra, Phys. Lett. 157B, 379 (1985).

[46] A. Strominger, Phys. Lett. B 421, 139 (1998).

[47] L. Andrianopoli et al., Nucl. Phys. B476, 397 (1996).

[48] J. Polchinski and A. Strominger, Phys. Lett. B 388, 736 (1996).

[49] J. Michelson, Nucl. Phys. B495, 127 (1997).

[50] P. M. Cowdall and P. K. Townsend, Phys. Lett. B 429, 281 (1998).

[51] E. Bergshoeff, M. de Roo, M. B. Green, G. Papadopoulos and P. K. Townsend, Nucl. Phys. B470, 113 (1996).

[52] L. J. Romans, Phys. Lett. 169B, 374 (1986).

[53] H. Lu, C. N. Pope, E. Sezgin and K. S. Stelle, Phys. Lett. B 371, 46 (1996); P. M. Cowdall, H. Lu, C. N. Pope, K. S. Stelle and P. K. Townsend, Nucl. Phys. B486, 49 (1997). 